\title{
CONTABILIDAD ADMINISTRATIVA. HERRAMIENTA PARA LA TOMA DE DECISIONES GERENCIALES.
}

\author{
Flor Isabel Salazar Vázquez ${ }^{1}$ \\ https://orcid.org/0000-0002-0071-0139 \\ Jorge Herminio Gonzalez Urgiles ${ }^{2}$ \\ https://orcid.org/0000-0001-7020-1260 \\ Paola Margoth Sanchez Salazar ${ }^{3}$ \\ https://orcid.org/0000-0002-3024-2962 \\ Jonnathan Fabián Sanmartin Gonzalez ${ }^{4}$ \\ https://orcid.org/0000-0002-8580-2277
}

\section{RESUMEN}

Las organizaciones requieren información contable, pertinente y oportuna para apoyar sus decisiones, lo que exige disponer de registros contables que provean información sobre la contabilización de los procesos clave. En este sentido, el objetivo del trabajo se centra en analizar la contabilidad administrativa, como una herramienta fundamental para ofrecer al tomador de decisiones, información ajustada a sus necesidades. Para ello se ha hecho una revisión bibliográfica desde diferentes perspectivas, a saber: Evia (2005), Garrison et al. (2007) y Chacón (2007), entre otros. La metodología empleada es de tipo documental-descriptiva, para ello fue necesario revisar fuentes especializadas en el tema, las mismas fueron analizadas desde su contenido, permitiendo establecer relaciones entre datos para generar información extrayendo de cada uno de ellos aspectos relevantes sobre la contabilidad. Entre los resultados obtenidos, resalta la importancia de la contabilidad administrativa, la cual apoyada en la contabilidad de costos es capaz de ofrecer información contable con gran valor para apoyar el proceso de toma decisiones. Es importante, disponer de registros, clasificar y organizar la información en base a los requerimientos reales de las organizaciones, su especificidad y naturaleza, con el fin de obtener información veraz sobre operaciones y transacciones realizadas por las mismas, siendo esto la base sobre la cual se puedan tomar decisiones gerenciales asertivas y oportunas.

Palabras clave: Contabilidad administrativa; contabilidad de costos; herramienta; toma de decisiones; organizaciones.

\section{Contabilidade administrativa. Ferramenta para faz̧er decisões gerenciais}

\section{RESUMO}

As organizações exigem informações contáveis, relevantes e oportunas para apoiar as suas decisões, o que exige ter registos contáveis que forneçam informações sobre a contabilidade dos principais processos. Nesse sentido, o objetivo do trabalho é focar na análise da contabilidade administrativa, como ferramenta fundamental para oferecer, aos tomadores de decisão, informações ajustadas às suas reais necessidades. Para isso, foi feita uma revisão bibliográfica sob diferentes perspectivas, a saber: Evia (2005), Garrison et al (2007) e Chacón, (2007), entre outras. A metodologia utilizada é do tipo documental-descritiva, para isso foi necessário revisar fontes especializadas sobre o assunto, elas foram analisadas a partir de seu conteúdo, possibilitando o estabelecimento de relações entre os dados, para gerar informações, extraindo aspectos relevantes da contabilidade de cada uma delas. Entre os resultados obtidos, destacase a importância da contabilidade administrativa, que, apoiada na contabilidade de custos, é capaz de oferecer informações contáveis, de grande valor, para subsidiar o processo de tomada de decisão. É importante ter registos, classificar e organizar as informações com base nos requisitos reais das organizações, em sua especificidade e natureza, a fim de obter informações verdadeiras sobre as operações e transações realizadas por elas, sendo esta a base sobre a qual decisões de gestão assertivas e oportunas podem ser tomadas.

Palavras-chave: Contabilidade administrativa; contabilidade de custos; ferramenta; tomada de decisão; organizações.

\footnotetext{
${ }^{1}$ Ingeniera empresarial, Máster en Auditoría Integral, Máster en Docencia Superior. Docente e investigador de la Universidad Católica de Cuenca, Ecuador. E-mail: fisalazarv@ucacue.edu.ec

2 Economista, Magíster en Auditoría Integral. Docente e investigador de la Universidad Católica de Cuenca, Ecuador. E-mail: igonzalezu@,ucacue.edu.ec

${ }^{3}$ Estudiante. de la Universidad Católica de Cuenca, Ecuador. E-mail: pmsanchezs93@est.ucacue.edu.ec

${ }^{4}$ Abogado. Libre ejercicio profesional, Ecuador. E-mail: walter.ms@hotmail.com; ihonnathan71@live.com
} 


\section{Administrative accounting. Tool for Managerial decision making}

\section{ABSTRACT}

Organizations require timely, relevant and accounting information to support their decisions, which requires having accounting records that provide information on accounting for key processes. In this sense, the objective of the work is focused on analyzing administrative accounting, as a fundamental tool to offer decision makers information adjusted to their real needs. For this, a bibliographic review has been made from different perspectives, namely: Evia (2005), Garrison et al. (2007) and Chacón, (2007), among others. The methodology is documentary-descriptive, specialized sources on the subject were reviewed, they were analyzed from their content, allowing relationships to be established between data to generate information, extracting relevant aspects of accounting from each of them. Among the results obtained, the importance of administrative accounting stands out, which, supported by cost accounting, is capable of offering accounting information with great value to support the decision-making process. It is important to have records, classify and organize the information based on the real requirements of the organizations, their specificity and nature, in order to obtain truthful information on operations and transactions carried out by them, this being the basis on which assertive and timely management decisions can be made.

Key words: Administrative accounting; cost accounting; tool; decision making; organizations.

\section{Introducción}

Una de las limitaciones más importantes a nivel de las organizaciones, es que en muchas oportunidades descartan la utilización de la información financiera, como la base para la toma de decisiones, perdiendo de vista los datos generados que se constituyen en insumos para la construcción de información y apoyar decisiones tanto estratégicas como operativas en las empresas. En tal sentido, disponer de registros precisos y organizados sobre las operaciones productivas, contables y transacciones económico-financiera, es un recurso valioso en las empresas, pues provee datos de interés para que gerentes en cualquier nivel de la estructura organizativa, tomen decisiones partiendo de la evaluación objetiva del desempeño de sus operaciones.

Un análisis integral de la situación de cualquier empresa, abarca un proceso complejo, donde la toma de decisiones se constituye en factor esencial que inicia con el despliegue de múltiples alternativas, las cuales deben ser evaluadas, optando por aquella que mayor beneficio provea para la organización. Esto exige disponer de datos e información cualitativa y cuantitativa, para apoyar las decisiones, orientadas a la asertividad.

Desde una perspectiva amplia, la contabilidad general o financiera, proporciona información para usuarios externos, representados por inversionistas, instituciones del gobierno, instituciones financieras, entre otros, mientras que la contabilidad administrativa genera información para que usuarios internos, tomen decisiones sobre la base de las operaciones realizadas (Garrison et al., 2007; Molina de Paredes, 2003; Ott, 1998; Evia, 2005; Jiambalvo, 2003; Hansen y Mowen, 2007; Polimeni et al., 2006). Es decir, registra, controla y reporta datos útiles a los gerentes en la planeación, control y toma de decisiones.

En función de ello, asumir la contabilidad administrativa como una opción en las empresas, exige una perspectiva orientada hacia el emprendimiento de prácticas gerenciales acordes con la realidad del negocio, a la vez que plantea la necesidad de disponer de sistemas de información contables, como medio para tomar decisiones. Así, la contabilidad administrativa, apoyada en la contabilidad de costos, permitirá una gestión adecuada de las operaciones contables desde la perspectiva de los costos, con visiones detalladas sobre las operaciones productivas y/o de prestación de servicios. En consideración de lo anterior, esta investigación se plantea como objetivo central analizar la contabilidad administrativa, asumiéndola como una herramienta fundamental para ofrecer al tomador de decisiones información ajustada a sus necesidades. A partir del empleo de esta herramienta, se genera información precisa, oportuna y detallada de interés para la gestión directiva de las empresas.

Desde el punto de vista metodológico, se plantea una investigación de tipo documental-descriptiva, detallando características de la contabilidad administrativa como objeto de estudio sobre el cual se establecen elementos esenciales como base para análisis futuros de otros fenómenos de mayor 
nivel de profundización. En este trabajo se empleó el análisis de contenido porque es un tipo de medición de carácter científico en el ámbito de las ciencias económicas y sociales, que permite establecer relaciones entre datos para generar información. Para Berelson y Lazarfeld (1948) y Lazarsfeld (1972) es una técnica de investigación, que "permite manejar un gran volumen de información a un costo no muy alto, lo que la hace especialmente útil para su utilización (...)" (Tinto, 2013, p. 13).

Los estudios descriptivos, permiten la descomposición del todo en partes, valiéndose del análisis que permita la comprensión e interpretación de la naturaleza actual de fenómenos particulares, para ello, la investigación se apoya en la revisión de documentos, entre los que resaltan textos y documentos científicos especializados en el área objeto de estudio; estos permiten sustentar la contabilidad administrativa, la cual partiendo del reconocimiento de variables exógenas (entornas a ella), y de elementos o aspectos internos, genera reportes e información sobre procesos medulares del negocio. Dicha información, se considera específica, detallada y de gran valor para la empresa; orienta la toma de decisiones, y sin duda define el direccionamiento futuro de la organización, permitiendo replantear de ser necesario, los procesos de planificación y control; aspectos que serán abordados en los siguientes apartados del trabajo, desde el entorno empresarial, posteriormente en su fundamentación, así como en el espacio que hace referencia a la toma de decisiones.

\section{Entorno empresarial y su relación con la contabilidad administrativa}

Durante las dos últimas décadas, los cambios suscitados en el entorno externo a las organizaciones, han generado conmoción en las empresas, así como fuertes impulsos para romper paradigmas asociados con formas tradicionales de hacer negocios. El crecimiento de la informática y las telecomunicaciones, así como de la competencia de los mercados, exige competitividad, calidad, innovación, y a la vez costos racionales y precios competitivos a nivel de los productos y servicios ofrecidos, resaltando el valor y apostando al lograr el complimiento de los objetivos y misiones establecidas.

Lo anterior requiere del reconocimiento pleno del entorno, para sobre esa base, definir estrategias que se adapten a los requerimientos externos ante la incertidumbre y presión que implica tomar decisiones rápidamente (Evia, 2005), estando conscientes que los cambios emprendidos en el ámbito externo repercuten de manera directa en la contabilidad administrativa. En este sentido, estrategias como el justo a tiempo, calidad total, reingeniería, producción ajustada, seis Sigma, entre otras exigen incrementar la calidad, la producción y las utilidades, a la vez que implican la reducción de costos y demoras asociadas al desarrollo de procesos fundamentales (Garrison et al, 2007; Molina de Paredes, 2003).

En todo caso, las estrategias adoptadas dependerán de factores políticos, económicos, legales, tecnológicos, demográficos, culturales, ambientales, sociales presentes en el entorno externo inmediato a la organización (Davis, 2003; Thompson y Strickland, 2006). Estos influyen directamente en el entorno interno, a la vez que exigen delinear estrategias oportunas que garanticen la permanencia de las empresas en mercados dinámicos, cambiantes y altamente globalizados y competitivos, donde la única constante es el cambio, y la asertiva respuesta que puedan dar los gerentes a las exigencias de la realidad que los circunda.

Sobre este particular, establecen Ott (1998) y Alturo y Dussan (2016) que la contabilidad de gestión es la encargada de suministrar las informaciones requeridas por los gestores, para llevar a cabo su tarea directiva y para atender a estos requerimientos; sin embargo, necesita desmarcarse del ámbito exclusivamente interno de la organización, y profundizar en la parte externa para adoptar una dimensión estratégica.

Así, aspectos externos, conjuntamente con elementos internos permiten conducir esfuerzos, que de la mano con el conocimiento pleno de aspectos contables y financieros son cruciales para la 
generación de ventajas competitivas, orientadas a propiciar la diferenciación, calidad, celeridad, precios y costos de manera sostenible para la organización.

También es de interés el conocimiento, no solo por parte de agentes externos de la información contable de la empresa, sino también la generación de información interna relativa a las operaciones medulares de la empresa; ésta, coadyuva o soporta las decisiones tomadas y permite afrontar retos, riesgos y la incertidumbre presente, a nivel mundial y en particular en economías en desarrollo, características de la realidad latinoamericana con mayor confianza y seguridad.

Conscientes de las oportunidades que pudieran existir en el entorno, pero también de las amenazas constantes, y de las exigencias de disponer información organizada, veraz y oportuna como fortalezas de la gestión, la contabilidad administrativa juega un rol fundamental en pequeñas, medianas o grandes organizaciones, en las que el soporte contable, desde una perspectiva gerencial, se torna esencial para las empresas.

A pesar de esta afirmación, no todas las organizaciones logran comprender esta realidad, ni las asociaciones existentes entre los aspectos antes comentados, y siguen manejando visiones restringidas del negocio, y de la importancia de disponer de información y conocimiento estratégico y útil, como parte de sus labores administrativas y contables.

Superar estas visiones, comenzar a interpretar, reconocer el entorno, así como cada uno de los factores o elementos que hacen parte de él, se considera fundamental. Las empresas, más aún las pequeñas y medianas, deben definir su direccionamiento, y reconocer la necesidad de disponer de información administrativa-contable sobre la que, gerentes y accionistas, soporten sus decisiones, y el desarrollo mismo de funciones como la planeación y el control de las operaciones.

Según plantea Evia (2005), los administradores tienen la posibilidad de apoyarse en el uso de herramientas y técnicas que les permitan utilizar sus sistemas de costos como herramientas estratégicas. Basado en esto, la contabilidad administrativa o de gestión no debe desligarse del entorno que rodea la organización pues cada una de las decisiones tomadas en el seno de la empresa, se encuentra influenciada por dicho contexto, caracterizado por oportunidades y restricciones que se deben identificar para poder manejar incertidumbre, riesgos y la dinamicidad presente en economías modernas.

\section{Aspectos fundamentales sobre la Contabilidad Administrativa}

Mucho se ha discutido acerca de lo que se entiende por contabilidad. En nuestros días, la contabilidad se conceptualiza como un sistema de procedimientos de registro de las consecuencias económicas de las operaciones que realiza una empresa (Evia, 2005). Para el autor, la gran mayoría de las operaciones que realiza una entidad (de cualquier tipo) son susceptibles de ser cuantificadas en términos económicos $\mathrm{y}$, por ende, originan modificaciones en la situación financiera $\mathrm{y}$ patrimonial de las organizaciones.

Por ello, la contabilidad como disciplina técnica, ofrece información sobre las transacciones y movimientos económicos de una organización. Se ocupa del estudio de estas transacciones y su relación con el mundo exterior, con el objeto de mostrar una visión global del comportamiento de una empresa, mediante instrumentos básicos como el balance general y el estado de ganancias y pérdidas (Chacón, 2007).

La contabilidad general o financiera, es de carácter obligatorio para las empresas. Provee información básica para accionistas, acreedores entre otros actores externos a la organización (Garrison et al., 2007), ofreciendo información sobre excedentes económicos globales de la empresa. Mientras que la contabilidad administrativa ofrece información pertinente orientada a la resolución de problemas y a la toma de decisiones.

La contabilidad administrativa, no está sujeta, a ningún tipo de normatividad (Evia, 2005); es flexible y libre (Garrison et al., 2007) y define criterios objetivos que le permiten ofrecer reportes detallados sobre el sector en el que opera la organización, sus productos, servicios, clientes, empleado, es decir realiza evaluaciones del negocio, proporcionando informes directivos que 
coadyuvan en el direccionamiento del negocio y en el control y evaluación del desempeño del mismo. Según Evia (2005), los sistemas de contabilidad administrativa pueden ser creados a la medida de las organizaciones, respondiendo a las demandas específicas de cada una en particular. La contabilidad administrativa, supera la visión tradicional manejada por la contabilidad financiera, orientando la información generada al soporte de decisiones estratégicas y operativas en las empresas. Busca proporcionar una visión integral de las modificaciones sufridas en el volumen y composición del patrimonio de una entidad a lo largo de un determinado período, como consecuencia de las transacciones que la misma lleve a cabo y de los imponderables a los que esté sujeta (Evia, 2005).

En otro orden de ideas, haciendo un poco de historia, se plantea que con el transcurrir de los años el término de contabilidad administrativa ha sido desplazado por el término contabilidad de gestión, enfatizando conceptos y procedimientos contables vitales para la elaboración de reportes destinados a usuarios internos de las organizaciones.

En ambos casos, sea la contabilidad administrativa o la contabilidad de gestión, proporcionan a gerentes información para planear, controlar y tomar decisiones (Jiambalvo, 2003). Según el autor, desde la perspectiva de la planeación, establece planes financieros denominados presupuestos, mientras que desde la perspectiva del control, evalúa continuamente el rendimiento de las operaciones productivas, para sobre esta base, tomar decisiones como parte integral del proceso de planeación y control. Es decir, se dedica al estudio de las transacciones que tienen lugar en el interior de las organizaciones, específicamente, las relacionadas con el núcleo de operaciones donde se llevan a cabo actividades de transformación de insumos en bienes y/o servicios (Chacón, 2007).

En función de lo anterior, se presenta en el cuadro 1, diferencias precisas manifiestas entre la contabilidad de gestión y la contabilidad administrativa, para, a partir de estos aspectos, se puedan manejar visiones específicas y detalladas sobre cada una de ellas, complementarias pero diferentes entre sí. Según Hansen y Mowen (2007), el sistema de información contable en una organización está constituido tanto por la contabilidad financiera, como por la contabilidad administrativa, ambos subsistemas importantes en una organización.

\section{Cuadro $\mathrm{N}^{\circ} 1$}

Diferencias entre la contabilidad general y la contabilidad administrativa

\begin{tabular}{|c|l|}
\hline \multicolumn{1}{|c|}{ Contabilidad General } & \multicolumn{1}{|c|}{ Contabilidad Administrativa } \\
\hline \multicolumn{1}{|c|}{ Caránter obligatorio } & \multicolumn{1}{|c|}{ Visión orientada a la toma de decisiones } \\
\hline \multicolumn{1}{|c|}{$\begin{array}{c}\text { Es opcional, pero estratégica en la toma de } \\
\text { decisiones }\end{array}$} \\
\hline $\begin{array}{l}\text { Desarrolla transacciones y movimientos } \\
\text { económicos de la empresa con el entorno externo. }\end{array}$ & $\begin{array}{l}\text { Desarrolla transacciones que tienen lugar en el } \\
\text { interior de las organizaciones. }\end{array}$ \\
\hline $\begin{array}{l}\text { Muestra a accionistas, acreedores y otros actores } \\
\text { externos una visión global del comportamiento de } \\
\text { una empresa mediante instrumentos básicos } \\
\text { (balance general - estado de ganancias y pérdidas). }\end{array}$ & $\begin{array}{l}\text { Ofrecer reportes detallados destinados a usuarios } \\
\text { internos de la organización sobre el sector en el } \\
\text { que opera, sus productos, servicios, clientes, } \\
\text { empleado, útiles en el direccionamiento del } \\
\text { negocio. }\end{array}$ \\
\hline $\begin{array}{l}\text { Ofrece información general, a especie de fotografía } \\
\text { de una empresa en un momento determinado. }\end{array}$ & $\begin{array}{l}\text { Ofrece información orientada a la resolución de } \\
\text { problemas, planear, controlar y tomar decisiones. }\end{array}$ \\
\hline $\begin{array}{l}\text { Se pliega a los principios de contabilidad } \\
\text { generalmente aceptados. }\end{array}$ & $\begin{array}{l}\text { Es flexible y libre. No se apega a principios, ni } \\
\text { normas rígida. }\end{array}$ \\
\hline
\end{tabular}

Fuente: Elaboración propia.

Para Lorino (1993), la contabilidad financiera presenta limitaciones que impiden satisfacer las necesidades en la gestión, razón por la cual las organizaciones deben apuntar al diseño de sistemas de contabilidad de gestión adaptados a los requerimientos de sus actividades, y esto lo ofrece la 
contabilidad administrativa, es específica y detallada; ofrece datos e información sobre la cual se respaldan decisiones importantes y estratégicas para las organizaciones.

Bajo estos planteamientos, Hansen y Mowen (2007) y Ponce y William (2017) y en función de la disertación realizada previamente sobre la contabilidad financiera y la administrativa, es pertinente plantear la necesidad de incorporar al sistema de información contable, la contabilidad de costos, como sistema híbrido que se desarrolla entre la contabilidad financiera y la contabilidad administrativa. La contabilidad de costos, ofrece información sobre la utilización de los costos de una compañía con fines internos y externos.

Sobre este particular, Polimeni et al (2006) definen a la contabilidad de costos como una técnica o método para determinar el costo de un proyecto, proceso o producto, teniendo como principal función, asignar, acumular, registrar y analizar información importante para el uso interno de los gerentes en la toma de decisiones. Por su parte, Pabón (2010) la refiere como una herramienta que permite un proceso eficiente, de análisis e interpretación de la información sobre los costos de producción, representando un factor clave en la gestión de las empresas manufactureras; mientras que Sinisterra (2007) la concibe como una técnica o mecánica contable que permite calcular el costo de fabricar un producto o prestar un servicio.

Por ello, la contabilidad administrativa en aras de tomar decisiones, se apoya en la contabilidad de costos, pues es esta la que considera todos los detalles referentes a los costos totales de producción, para posteriormente poder considerar el costo unitario del productos y servicios, y proporcionar información relevante con la finalidad de evaluar y controlar la actividad productiva o de prestación de servicios.

Los costos totales, contienen elementos básicos para la determinación del costo de un producto (Sinisterra, 2007), y están representados por: 1) materiales directos, definidos como la materia prima o insumos que una vez sometidos a un proceso de transformación, se convierten en productos terminados, es decir terminarán siendo parte del producto final; 2) mano de obra directa, representado como el esfuerzo físico que aportan los trabajadores relacionados con el proceso productivo, sea por acción manual u operando una máquina, este costo es fácilmente identificable con la producción y se puede rastrear de forma directa; y 3) costos indirectos de fabricación (CIF), se constituyen en costos utilizados para acumular los materiales indirectos, la mano de obra indirecta y los demás costos indirectos de fabricación, que no pueden identificarse plena y directamente con los productos específicos (Polimeni et al., 2006; Backer et al., 2000; Hansen y Mowen, 2007; Gayle, 1999; Horngren et al., 2009; Santos, 2008).

Asimismo, la contabilidad de costos se vale de sistemas de información y metodologías de costeo que permiten concretar datos precisos de costos para los productos y servicios que se fabrican o prestan en las organizaciones. Estos permiten el registro, identificación, clasificación, acumulación, análisis e interpretación de la información de costos, con el propósito de proveer informes valiosos a la gerencia, que permitan la toma de decisiones sobre la planeación y control de las operaciones, evaluación del desempeño, utilización de recursos, definición e implementación de estrategias, elaboración de planes, fijación de políticas, entre otros (Pabón, 2010).

Tanto la acumulación y clasificación de los costos de producción, representan tareas que demandan tiempo y dedicación, por la cantidad de datos que los soportan y la recolección organizada que exige de datos de costos (Polimeni et al., 2006), sin embargo, son necesarias para suministrar información para la toma de decisiones, y en vista de que la determinación del costo total de producción suministra poca información acerca de las operaciones de una empresa, los sistemas de costos permiten la determinación de los costos unitarios de producción, como fuente de información clave para la toma de decisiones asertivas.

Los sistemas de costos ofrecen al gerente de costos, normas contables, técnicas y procedimientos de acumulación de datos de costos, con el propósito de determinar el costo unitario del producto fabricado, planear los costos de producción y contribuir con la toma de decisiones (Sinisterra, 2007). Representan herramientas para generar información precisa sobre los costos de producción 
y la forma de proceder en cuanto a actividades, procesos, productos, servicios y clientes de la organización (Kaplan y Cooper, 2003). Según los autores, las empresas utilizan los sistemas de costos para alcanzar objetivos como: diseñar productos y servicios que satisfagan las expectativas de los clientes; realizar mejores continuas o reingeniería de calidad, eficiencia o rapidez; guiar las decisiones de inversión; negociar con clientes precio, características del producto, calidad, condiciones de entrega, entre otros aspectos; estructurar procesos eficientes de distribución para los clientes, entre otros.

Basado en los argumentos anteriores, se puede afirmar que la contabilidad de gestión se apoya en la contabilidad de costos, siendo esta ultima la que indica a la dirección de la empresa las consecuencias de la realización de una actividad o la no realización de la misma (Ortega, 1999); calcula eficiencias, costos y rendimientos, agregando valor a la información ofrecida por la contabilidad financiera (cálculo de beneficios y valor patrimonial de la organización).

Como sistema de información, la contabilidad de costos se centra en la determinación de los costos de los productos, relacionándolos con los ingresos obtenidos por la venta de los mismos, y de esta forma genera información para la toma de decisiones (Rincón, 2005). De allí que el objetivo central sea facilitar a la dirección de las organizaciones información útil para la toma de decisiones económicas, y dar respuestas a las necesidades planteadas en el seno de la organización, necesarias para la gestión (costos, rentabilidad e indicadores no monetarios), concentrándose en dirigir los esfuerzos de la organización hacia la consecución de los objetivos y hacia la procura de eficiencia y la eficacia en la utilización de recursos (Balcázar et al., 2019)(Amat y Soldevilla, 2000).

Sintetizando, la contabilidad de costos, se reconoce la como un área de la contabilidad que ha superado limitaciones identificadas por gerentes, en la contabilidad legal, financiera o tradicional, buscando satisfacer exigencias de organizaciones nacientes. Así, mientras la contabilidad de gestión incorpora elementos de gestión como la planificación, el control y la toma de decisiones, la contabilidad de costos, es parte de ella, y ofrece información útil para la reformulación de planes, el establecimiento de mecanismos de control y sin duda, para sustentar decisiones organizacionales. En este contexto, tanto la contabilidad de costos como la contabilidad de gestión, e incluso la denominada contabilidad de dirección estratégica, pretenden responder a las necesidades de registro, control e información planteadas dentro de las organizaciones con el fin de optimizar sus costos, ser competitivas y garantizar su supervivencia (ver figura 1).

\section{Figura $\mathrm{N}^{\circ} 1$}

Contabilidad financiera - contabilidad administrativa - Contabilidad de costos

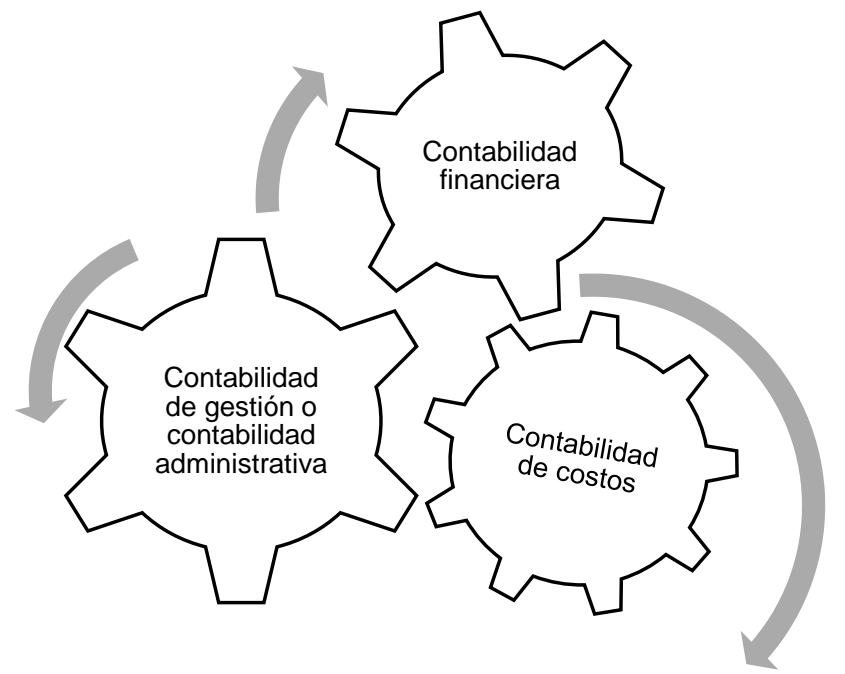

Fuente: Elaboración propia. 


\section{Toma de decisiones: elementos esenciales}

Las organizaciones insertas en contextos altamente dinámicos y cambiantes, deben constantemente revisar y redefinir estrategias como medios o acciones que le permitan afrontar los continuos desafíos que se le presenten. Por ello, el proceso de toma de decisiones gerenciales, representan una necesidad para el gerente de evitar la incertidumbre que genera el difícil acto de decidir (Arrendondo y Vázquez, 2013).

El hecho de seleccionar entre múltiples alternativas o cursos de acción, debe estar apoyado en modelos y metodologías que permitan a los niveles decisorios, disponer de información sobre la cual puedan sustentar sus decisiones. En este sentido, si se combina la influencia que tienen factores del entorno externo a las organizaciones, con aspectos inherentes a ella es posible que unos determinen los otros, y definan las decisiones a tomar en la empresa. Esta dependencia crítica que existe entre la organización y su medio ambiente externo, llevan a que las organizaciones estén siempre alerta a los cambios que se generan en él y, por consiguiente, que dispongan de un conocimiento previo de cómo se conforma y comporta el medio ambiente (González y Bermúdez, 2008), y sobre esta base organizar información a lo interno de sus empresas y generar información precisa para afrontar los desafíos que se presentan en su transitar.

$\mathrm{Al}$ respecto, la contabilidad administrativa mediante "(...) reportes o informes, sirven para tomar decisiones financieras oportunas, tales como ver si la implementación de un nuevo producto o servicio es rentable, o si comprar el mismo producto y comercializarlo es la mejor opción" (González, 2016, p.14), para ello han surgido enfoques como: relación costo-beneficio, correlaciones técnicas-comporamiento, así como reconsiderar la estructura de costos, entre otros. Así, decisiones sobre nuevos productos, ampliación de líneas de producción, establecimiento de precios, incremento de la capacidad de producción, nuevas inversiones, establecimiento de alianzas con otras empresas, formulación y evaluación de proyectos especiales, selección de cursos alternativos de acción, toma de decisiones sobre inversión de capital y otros proyectos orientados al crecimiento de la empresa entre otras, deben estar apoyadas en datos e información cuantitativa generada en el seno de la empresa.

Asociando el proceso decisorio con la contabilidad administrativa o de gestión, plantea Esteban (S/F), que la contabilidad administrativa, pretende cubrir las limitaciones de la información financiera ante determinados aspectos clave, fundamentales en el proceso de toma de decisiones, siendo esta área de la organización, una de las principales proveedoras de información para la dirección en la toma de decisiones que permite tener el control de todas las operaciones de la empresa, ya que el gerente de costos, buscará generar información pertinente para apoyar sus decisiones, a partir de la consideración de la mayor cantidad de elementos posibles, pues de esta manera no solo evitará la irracionalidad de sus acciones, sino también, alcanzará mayor objetividad y certeza de las mismas (Arrendondo y Vázquez, 2013) (Sifontes et al., 2015).

En este sentido, no solo es de importancia la experiencia de quien toma las decisiones (elementos cualitativos), sino la interpretación de la información económica-financiera de la que disponga la empresa. De su calidad, oportunidad y veracidad dependerá el éxito de la organización en el futuro. Por ello, es fundamental considerar en el proceso de toma de decisiones las alternativas existentes o cursos de acción sobre los cuales se erige una decisión. Disponer de información contable y de los costos asociados a cada una de las actividades, procesos y áreas de la organización se torna fundamental para apoyar decisiones organizacionales.

Según Franklin (2011), tanto los individuos como las organizaciones prosperan o fracasan como resultado de las decisiones que toman, razón por la cual, los responsables de tomar las decisiones, deben centrarse en información de tiempo real e involucrar expertos en el proceso de toma de decisiones. De allí que, la integración de la información que ofrece la contabilidad administrativa, apoyada en la contabilidad de costos se torna crucial para soportar decisiones operativas y estratégicas de empresas en particular que se someten a contextos altamente exigentes y demandantes, donde deben luchar por permanecer y lograr posiciones de domino y liderazgo. 


\section{Contabilidad Administrativa como Herramienta para la Toma de Decisiones}

En espacios anteriores se ha hecho un esbozo de la contabilidad administrativa, así como sus bondades en las organizaciones, dejando claro que “(...) se basa en un razonamiento económico inductivo que ha sido perfeccionado constantemente desde varias perspectivas (...)" (Giménez et al., 2015, p. 209); sin embargo, resulta oportuno en este momento especificar que ella ofrece "lineamientos que ayudan a los contadores administrativos a proveer el valor máximo a sus compañías en la toma de decisiones estratégicas y operacionales: el enfoque de costo-beneficio, consideraciones técnicas y de comportamiento y diferentes costos para distintos propósitos" (Horngren et al., 2012, p.11), y a partir de esos lineamientos se toman decisiones, utilizando el método científico; el cual constituyen en seis actividades, a saber: definir el problema, identificar alternativas, identificar los costos y beneficios de cada alternativa factible, considerar factores cualitativos y cuantitativos, seleccionar la estrategia que ofrezca mayor beneficio y monitorear la alternativa seleccionada. Las decisiones que se toman están acopladas con la estrategia organizacional, ello permite la coordinación en diferentes direcciones y resultados consistentes, así como "proyectar nuevas tendencias, transformar y elaborar juicios para que los procesos administrativos sean efectivos" (Barzola, 2018, p. 19). Explica Ramírez (2013) que el empleo del método científico permite especificar en la tercera y cuarta actividad que la contabilidad administrativa coadyuva a que las decisiones que tomen sean más asertivas, pues facilita información más precisa.

De allí que la contabilidad administrativa tiene un rol importante en la calidad de la información, facilitando la construcción de escenarios y sus análisis desde diferentes perspectivas, según el tipo de industria y actividad que se trate, para tomar decisiones en congruencia con los objetivos y estrategias empresariales. En tal sentido, la vinculación de las decisiones que se toman con las estrategias orientara el direccionamiento empresarial hacia el beneficio de los accionistas en pro de resultados consistentes.

Así como orienta direccionamientos, también facilitan el control de los procesos, contrastar el desempeño real con el desempeño que se presupuestó e informando a la gerencia sobre su desempeño, el desempeño de sus subalternos, detectar puntos débiles con el fin de encontrar correctivos ir mejorando en cada aspecto para el desarrollo de la actividad de la empresa, así como resaltar aquellos indicadores de progreso y crecimiento para motivar a todos los involucrados a continuar su participación proactiva para alcanzar los objetivos y metas empresariales.

\section{Conclusiones}

En este trabajo se ha expuesto los elementos generales de la contabilidad financiera como área de la contabilidad general que antecede a la contabilidad administrativa o de gestión. Por un lado, la contabilidad general o financiera precisa información para agentes externos a la organización, mientras que por el otro, la contabilidad administrativa o de gestión que apoyada en la contabilidad de costos, ofrece información que alimenta informes internos de la organización.

Desde la óptica del detalle y la especificidad de las operaciones, la contabilidad administrativa cuantifica detalladamente transacciones operativas clave, a la vez que soporta las decisiones, direccionado las acciones hacia la asertividad y confiabilidad. Por lo que, ambas se complementan entre sí, generando reportes estratégicos para potenciar la competitividad y el direccionamiento del negocio en mercados dinámicos y altamente exigentes.

El disponer de registros de datos específicos y lograr su organización de forma sistemática y ordenada, permitirá generar información contable de calidad, oportuna y veraz sobre las operaciones desarrolladas y los recursos por estas consumidos. Por ello, la contabilidad administrativa se constituye en una herramienta de planeación y control, ambos elementos básicos del proceso administrativo, que proporcionan las bases para que los directivos de las empresas conduzcan sus procesos decisorios. 
Ante las exigencias de esta área de la contabilidad, se constituye en un reto el hecho de que las empresas puedan disponer de información detallada sobre sus operaciones productivas, en aras de optimizar sus procesos operativos y de toma de decisiones.

\section{Referencias bibliográficas}

Alturo, Carlos; Dussan, Carlos (2016). Contabilidad de gestión en la pequeña empresa manufacturera como herramienta estratégica para la competitividad. Un estudio desde El Espinal, Colombia. Revista Avances de Investigación. No. 11. (Pp.1-19) Extraído de http: https://repository.ucc.edu.co/bitstream/20.500.12494/13685/1/2016 WK Contabilidad $\% 20 \mathrm{de} \% 20 \mathrm{Gesti} \%$ c3\%b3n Abeiro Dussan.pdf

Amat, Oriol; Soldevilla, Pilar (2000). Contabilidad y Gestión de Costos. Tercera Edición. Editorial Gestión 2000. España.

Backer, Morton; Jacobsen, Lyle y Ramírez, David (2000). Contabilidad de costos. Un enfoque administrativo para la toma de decisiones. Editorial Mc Graw Hill Interamericana. México.

Balcázar, Bibiana; Narváez, Cecilia; Erazo, Juan (2019). Herramientas de contabilidad gerencial para la toma de decisiones financieras en la Empresa Tapitex M\&B Cía. Ltda. de la ciudad de Cuenca. Revista Visionario Digital. Vol 3. No. 22. Ecuador. (Pp. 50-80).

Barzola, María (2018). La contabilidad administrativa y su impacto en la toma de decisiones de las pymes comerciales. Trabajo de titulación de grado previo a la obtención del título de ingeniera en contaduría pública y aurditoría. Universidad Estatal de Milagro. Ecuador. Extraído

de http://repositorio.unemi.edu.ec/bitstream/123456789/4261/1/LA\%20CONTABILIDA D $\% 20$ ADMINISTRATIVA $\% 20 \mathrm{Y} \% 20$ SU $\% 20$ IMPACTO $\% 20$ EN $\% 20 L A \% 20$ TOMA $\% 20$ DE\%20DECISIONES\%20DE\%20LAS\%20PYMES\%20COMERCIALES.pdf.

Berelson, Bernard y Lazarfeld, Paul (1948) The analysis of communication content. University of Chicago and Columbia University, Estados Unidos.

Chacón, Galia (2007). La contabilidad de costos, los sistemas de control de gestión y la rentabilidad de la empresa. Actualidad Contable FACES Año 10 No $^{\circ}$ 15. Venezuela. (Pp. 29-45).

Davis, Fred (2003). Conceptos de Administración estratégica. Novena edición. Editorial Pearson Hall. México.

Esteban, Luisa (S/F). Contabilidad de gestión como herramienta para la toma de decisiones. Departamento de Contabilidad y finanzas, Universidad de Zaragoza. España

Evia, Manuel (2005). ¿Es la contabilidad administrativa una herramienta útil para desarrollar la competitividad de las empresas? Contaduría y Administración, núm. 219. México. (Pp.146-166).

Franklin, Enrique (2011). Toma de decisiones empresariales. Reseña de Comportamiento organizacional, enfoque para América Latina. Contabilidad y Negocios, vol. 6, núm. 11, julio, 2011. (Pp. 113-120). Extraído de http: http://www.redalyc.org/pdf/2816/281622820010.pdf

Garrison, Ray; Noreen, Eric; Brewer, Peter (2007). Contabilidad Administrativa. Editorial McGraw Hill. México.

Gayle, Letricia (1999). Contabilidad y administración de costos. 6ta edición. Editorial McGraw Hill Interamericana Editores, S.A. México.

Giménez, Daisis; Marín, Claudia y Cedeño, Sileyna (2015). Factores que influyen en el modelo de costos aplicados para el proceso de toma de decisiones gerenciales por las empresas hoteleras del Estado Nueva Esparta. PASOS. Revista de Turismo y Patrimonio Cultural. Vol. 13 No. 1. Venezuela (207-222). Extraído de https://doi.org/10.25145/i.pasos.2015.13.014.

González, Patricia y Bermúdez, Tatiana (2008). Una aproximación al modelo de toma de decisiones usado por los gerentes de las micro, pequeñas y medianas empresas ubicadas en 
Cali, Colombia desde un enfoque de modelos de decisión e indicadores financieros y no financieros. Revista Contaduría Universidad de Antioquia. No. 52. Colombia. (Pp. 131154).

González, José (2016). La contabilidad administrativa y la toma de decisiones financieras. Trabajo de titulación previo a la obtención del título de ingeniero en contabilidad y auditoría - CPA. Universidad Laica Vicente Rocafuerte de Guayaquil. Ecuador.

Hansen, Don y Mowen Maryanne (2007). Administración de costos. Contabilidad y control. Quinta Edición. Thompson Learning. México.

Horngren, Charles; Datar, Srikant y Foster, George (2009). Contabilidad de costos. Un enfoque gerencial. 12da edición. Editoral Pearson Prentice Hall. México.

Horngren, Charles; Datar, Srikant y Rajan, Madhav (2012). Contabilidad de costos. Un enfoque gerencial. 14ta edición. Editorial Pearson Educación, México.

Jiambalvo, James (2003). Contabilidad administrativa. Universidad de Washington Seatle. Editorial Limusa Wiley. México.

Kaplan, Robert y Cooper, Robin. (2003). Cortes \& Efecto. Editorial Gestion 2000. España.

Lazarsfeld, Paul. (1972). Qualitative Analysis. Boston: Allyn and Bac.

Lorino, Philippe (1993). El control de gestión estratégico. La gestión por actividades. Alfaomega Marcombo. Colombia.

Molina de Paredes, Olga Rosa (2003). Nuevas Técnicas de Control y Gestión de Costos en Búsqueda de la Competitividad. Actualidad Contable FACES. Año 6 No.6. Venezuela. (Pp. 25-32).

Ortega, Julio. (1999). Introducción a la contabilidad de gestión. Universidad Pontíficia. España.

Ott, Ernani (1998). La información contable y el proceso de toma de decisiones. Marco conceptual y estudio empírico. Tesis doctoral presentada en la Universidad de Deusto. España.

Pabón, Hernán (2010). Fundamentos de costos. Editorial Alfaomega Colombiana. Colombia.

Ponce, Viteri y Miriam, William (2017). Teoría de Estructura de Giddens en el análisis de la contabilidad gerencial. Revista publicando. 4. No. 11. Ecuador. (Pp. 658-667).

Polimeni, Ralph; Fabozzi, Frank y Adelberg, Arthur (2006). Contabilidad de costos. Conceptos y aplicaciones para la toma de decisiones gerenciales. Editorial McGraw-Hill Interamericana S.A. Colombia.

Ramírez, David (2013). Contabilidad administrativa. Un enfoque estratégico para competir. Editorial Mc Graw Hill Education. México.

Rincón, Haydée (2005). Contabilidad de costos y de gestión en la industria farmacéutica venezolana: estudio de un caso. Revista Venezolana de Gerencia. Vol. 10, núm. 30. (Pp. 267-287). Extraído de http: http://ve.scielo.org/scielo.php?script=sci arttext\&pid=S131599842005000200006\&lng $=$ es\&tlng $=$ es

Santos, Carló (2008). Contabilidad de costos. Costos predeterminados, Estimados y estándar. Tomo I Segunda Edición. Universidad del Zulia. Venezuela.

Sifontes, Daisis; Marín, Claudia; Cedeño, Sileyna (2015). Factores que influyen en el modelo de costos aplicados para el proceso de toma de decisiones gerenciales por las empresas hoteleras del Estado Nueva Esparta. Revista de Turismo y Patrimonio Cultural. Vol. 13 No, 1. (Pp. 207-222). Extraído de http:http://www.pasosonline.org/Publicados/13115/PS0115 14.pdf

Sinisterra, Gonzalo (2007). Contabilidad de costos. 2da edición. Editorial Ecoe Ediciones. Colombia.

Tinto, José. (2013). El análisis de contenido como herramienta de utilidad para la realización de una investigación descriptiva. Un ejemplo de aplicación práctica utilizado para conocer las 
investigaciones realizadas sobre la imagen de marca de España y el efecto país de origen.

Revista Provincia. Núm. 29. Venezuela. (Pp. 135-173).

Thompson, Arthur y Strickland, John (2006). Administración Estratégica. Undécima edición. McGraw-Hill. México. 